\title{
Quantitative 3D Characterization of Novel Polymer-nanocrystal Hybrid Catalysts by Electron Tomography
}

\author{
Andrew Herzing ${ }^{1}$, Andrew Riscoe ${ }^{2}$ and Matteo Cargnello ${ }^{2}$ \\ ${ }^{1}$ NIST, Gaithersburg, Maryland, United States, ${ }^{2}$ Stanford University, Stanford, California, United States
}

Biological enzymes are very effective catalysts which have evolved the capability to carry out chemical reactions at room temperature with high selectivity. Their diverse amino acid functional groups stabilize specific reaction intermediate states and control their transport to and from an active metal site at their center. However, they can only operate under highly specific reaction conditions, and designing a catalyst with similar behavior that can be used in industrial conditions is challenging. Recently, a novel class of catalysts was reported [1] which mimics this enzymatic functionality to produce highly selective, synthetic catalysts. These polymer-nanocrystal hybrid catalysts are produced in a modular fashion in which the first step is the production of a nanoporous polymeric organic framework (POF) to serve as the support structure. This is followed by impregnation of the support with a metal containing solution and a rapid calcination treatment to produce nanocrystals dispersed on the POF surface. Finally, the entire assembly is encapsulated in a second layer of POF material. The versatility of this type of catalyst is that the transport of reactants and products to and from the active metal sites can be controlled by varying the pore-size and thickness of the overlayer. The type of reaction intermediates that form can also be affected by changing the chemistry of the POF.

In order to better understand the catalytic performance, direct measurement of the metal nanoparticle distribution, including their distance from the surface and from each other, would be quite valuable. While the spatial resolution of transmission electron microscopy is more than sufficient for this task, individual projection images cannot quantitatively provide this information due to the complex, three-dimensional nature of the morphology. The catalyst ensembles are quite large (spanning microns) and exhibit a great deal of topographic variation as well as surface roughness.

To overcome these limitations, we have performed scanning transmission electron tomography analyses of a series of POF-encapsulated Pd nanoparticle catalysts with varying overlayer thicknesses. Annular dark-field (ADF) images were collected using a primary beam energy of $300 \mathrm{keV}$ and a small probeforming aperture $(10 \mu \mathrm{m})$ to limit the convergence semi-angle to less than $1 \mathrm{mrad}$. The resulting depth of focus was greater than $1.5 \mu \mathrm{m}$, such that whole catalyst ensembles could be imaged with all portions in focus simultaneously. ADF images (Fig. 1A) were collected over a tilt range of at least $\pm 65^{\circ}$. Images were spatially registered using the OpenCV implementation of phase correlation [2] followed by particle tracking for fine alignment [3] and reconstruction using the simultaneous iterative reconstruction technique in the ASTRA toolbox [4]. To extract the particle distribution metrics, a simple binary threshold was applied to the tomograms to isolate the individual nanoparticles from the support after which the centroid position was determined for each using the Python Scikit-image library [5]. The outer surface of the POF was located by applying the Otsu [7] threshold to the tomogram to produce a binarized volume of the entire ensemble and then using a Canny edge detection filter [6] to find the surface pixels. The nearest point on this surface was then calculated for each particle centroid to generate a histogram of particle-surface distances. This process was also applied to a single projection image for comparison between the two approaches. 
The results of the single projection analysis are shown graphically Fig. 1B along with a 3D depiction of the volumetric analysis of the same catalyst ensemble in Fig. 1C. The histogram comparison (Fig. 1D) of the two approaches clearly shows the advantage of the tomographic analysis. Whereas the values determined from a single projection show a large spread due to the topographic variation of the POF, those generated from the full tomographic analysis show a narrow, semi-Gaussian distribution with a mean particle-surface-distance of $(16 \pm 6) \mathrm{nm}$. The narrow spread of distances is consistent with that expected from the modular synthesis process, where the POF overlayer grows conformally to the underlying substrate. The quantitative assessment of these and other morphological metrics will be discussed in context with the catalytic performance observed as a function of overlayer thickness.
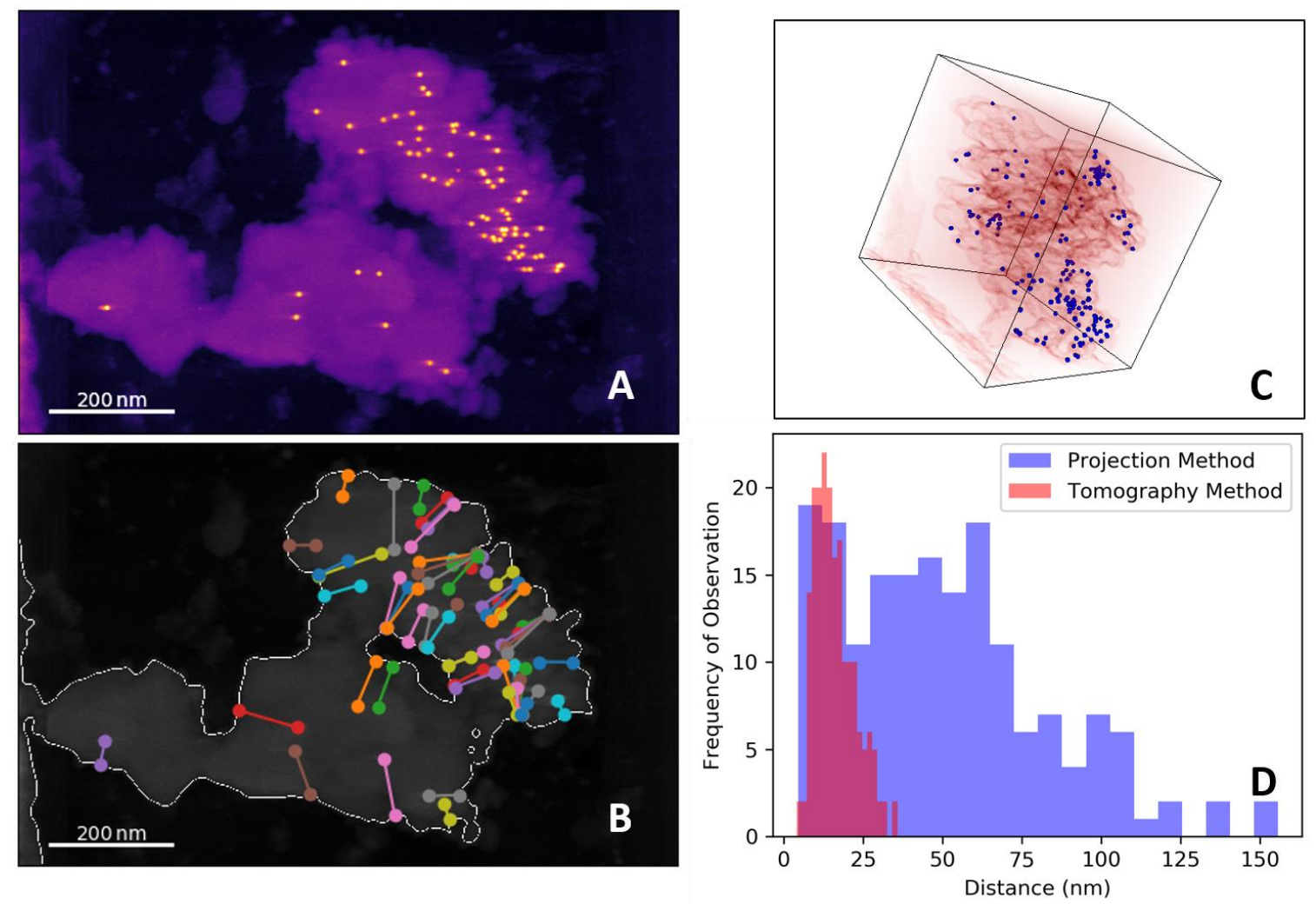

Figure 1. Single projection ADF-STEM image of a POF/Pd/POF encapsulated nanoparticle catalyst (A). Particle centroids and distances from overlayer surface (B) as measured from a single projection. 3D rendering $(\mathrm{C})$ of the POF surface location (red) and volumetrically determined particle centroids (blue). Histograms of particle-surface distances as determined from the single projection (blue) and from the full reconstructed volume.

References

1. A. R. Riscoe, A.R., Wrasman, C.J., Herzing, A.A. et al.Nat. Catal.2 (2019) 852-863.

2. G. Bradski. Dr. Dobb's J. Soft. Tools 5 (2000) 6.

3. F. Amat, et al. J. Struct. Bio., 161 (2008) 260.

4. W. van Aarle, et al. Ultramic., 157 (2015) 35.

5. Stéfan van der Walt, et al. PeerJ, 2 (2014) e453.

6. N. Otsu, IEEE Trans. Sys. Man. Cyber, 9(1979) 62.

7. J. A. Canny, IEEE Trans. Patt. Analy. Mach. Int., 8 (1986) 679. 